PROCEEDINGS OF THE

AMERICAN MATHEMATICAL SOCIETY

Volume 138, Number 5, May 2010, Pages 1621-1632

S 0002-9939(09)09483-0

Article electronically published on December 16, 2009

\title{
POSITIVE SCALAR CURVATURE OF TOTALLY NONSPIN MANIFOLDS
}

\author{
STANLEY CHANG \\ (Communicated by Alexander N. Dranishnikov)
}

\begin{abstract}
In this paper we address the issue of positive scalar curvature on oriented nonspin compact manifolds whose universal cover is also nonspin. We provide a conjecture for an obstruction to such curvature in this venue that takes into account all the data known to date. The conjecture is proved for a wide class of closed manifolds based on their fundamental group structure.
\end{abstract}

\section{INTRODUCTION}

The positive scalar curvature problem for spin manifolds has been extensively studied. For connected closed spin manifolds $M$ of dimension at least five and fundamental group $\Gamma$, the Gromov-Lawson-Rosenberg Conjecture predicted that the existence of such a metric on $M$ is equivalent to the vanishing of the generalized Dirac index $\alpha(M, f)$ in the real $K$-theory group $K O_{n}\left(C_{r}^{*} \Gamma\right)$. Here $f: M \rightarrow B \Gamma$ is the classifying map for the universal cover of $M$. While this conjecture is now known to be false for general $\Gamma$, a stable version of this conjecture definitively holds for spin manifolds with fundamental group $\Gamma$ provided that the Baum-Connes assembly map $K O_{*}^{\Gamma}(\underline{E}) \rightarrow K O_{*}\left(C^{*} \Gamma\right)$ is injective. In particular, the hypothesis of this theorem is satisfied whenever $\Gamma$ can be embedded discretely in a Lie group with finitely many connected components. For a survey on this subject, the reader should consult [10]. A discussion of the relationship between positive curvature and the Novikov conjecture can be found in [20, 21, 22.

In this article we will examine the nonspin case. Let $M^{n}$ be a connected closed manifold of dimension at least 5 and let $f: M \rightarrow B \Gamma$ be the classifying map for its universal cover. If $M$ is oriented, then we say that $M$ is totally nonspin if its universal cover $\widetilde{M}$ is nonspin. Note that a spin structure on $M$ lifts to a spin structure on the universal cover, so a totally nonspin manifold must itself be nonspin. Gromov and Lawson have shown that if $M^{n}$ is closed, simply connected and nonspin, then $M$ admits a metric of positive scalar curvature for $n \geq 5$. Along these lines, one can postulate that any closed, totally nonspin manifold $M^{n}$ with $n \geq 5$ has the same curvature phenomenon. While this statement holds for orientable manifolds with finite cyclic fundamental group, a counterexample to this conjecture has long been known. Schoen and Yau have shown using minimal hypersurface techniques

Received by the editors April 9, 2007, and, in revised form, January 8, 2008.

2010 Mathematics Subject Classification. Primary 32Q10.

Key words and phrases. Nonspin manifolds, proper classifying spaces, positive scalar curvature. This research was partially supported by NSF Grant DMS-9971657.

(C)2009 American Mathematical Society Reverts to public domain 28 years from publication 
that the totally nonspin manifold $T^{6} \#\left(\mathbb{C P}^{2} \times S^{2}\right)$ lacks any metric of positive scalar curvature.

Until now no obstruction to positive scalar curvature on totally nonspin manifolds has been identified or even proposed. We will attempt to repair this defect by examining the following conjectures, first stated by Rosenberg and Weinberger. Here for a finitely presented group $\Gamma$ we denote by $B \Gamma$ the usual classifying space for free $\Gamma$-actions and by $\underline{B} \Gamma$ the classifying space for proper $\Gamma$-actions. Notice that it is clear that Conjecture 2 follows immediately from Conjecture 1.

Conjecture 1. Suppose that $M$ is a totally nonspin manifold with fundamental group $\Gamma$ and dimension at least five. Let $f: M \rightarrow \underline{B} \Gamma$ be the composition of the classifying map $M \rightarrow B \Gamma$ and the natural map $B \Gamma \rightarrow \underline{B} \Gamma$. Denote by $[M]$ the fundamental class of $M$ in $H_{n}(M)$. Then $M$ admits a metric of positive scalar curvature iff $f_{*}[M]$ vanishes in $H_{n}(\underline{B} \Gamma)$.

Conjecture 2. If $\operatorname{dim} \underline{B} \Gamma<\infty$, then there is an integer $N$ such that if $M$ is a totally nonspin manifold with $\pi_{1}(M)=\Gamma$ and $\operatorname{dim} M>N$, then $M$ has a metric of positive scalar curvature.

Since totally nonspin manifolds without metrics of positive scalar curvature are not well understood and difficult to identify, we will mostly be offering positive solutions to Conjecture 1, with some notable exceptions in Section 4. It should be noted that results in the flavor of Corollary 2 appear in 3 and 9 .

The unstable Gromov-Lawson-Rosenberg conjecture, which was first shown to fail in the case $\Gamma=\mathbb{Z}^{4} \times \mathbb{Z}_{3}$, has nevertheless been verified for various cyclic groups, quaternionic groups, free groups, free abelian groups and the fundamental groups of orientable surfaces. It is now known that this conjecture also holds for groups $\Gamma$ of small geometric dimension, in particular if $\operatorname{dim}(M) \geq \max \{\operatorname{dim}(B \Gamma)-4,5\}$ provided that the assembly map for $\Gamma$ is split injective [11. Our goal is to verify the above conjectures for similarly large classes of groups. In particular, we will establish the validity of either or both of these statements for finite elementary groups, free abelian groups, maximally self-normalizing groups and groups satisfying various cohomological dimension conditions.

I would like to thank Shmuel Weinberger, Jim Davis and Jonathan Alperin for useful conversations. Special thanks go to the referee for offering very helpful comments and suggestions.

\section{Proper ClASSifying SPACES}

We describe the construction and some basic properties of $\underline{B} \Gamma$, the classifying space for proper $\Gamma$-actions [1, 16, 17. We will discuss them in some generality, although for the purposes of the theorems of this paper we will later require $\Gamma$ only to be discrete and finitely presented. To each locally compact group $\Gamma$, we can associate a space $\underline{E} \Gamma$ which is defined up to equivariant homotopy in the following manner. First, we say that a topological space $X$ is a $\Gamma$-space if $X$ is endowed with a continuous action $\Gamma \times X \rightarrow X$ of $\Gamma$ on $X$. Assume that both $X$ and $X / \Gamma$ are both metrizable. We say that the action is proper if, for every $p \in X$, there is a triple $(U, H, \rho)$ such that (a) $U$ is an open neighborhood of $p$ in $X$ with $g u \in U$ for all $(g, u) \in \Gamma \times U$, (b) $H$ is a compact subgroup of $\Gamma$, (c) the map $\rho: U \rightarrow \Gamma / H$ is continuous $\Gamma$-equivariant. In this case the space $X$ is called a proper $\Gamma$-space. 
A universal example for proper $\Gamma$-actions is a proper $\Gamma$-space $\underline{E} \Gamma$ such that if $X$ is any proper $\Gamma$-space, then there is a $\Gamma$-map $g: X \rightarrow \underline{E} \Gamma$ that is unique up to $\Gamma$-homotopy; i.e. any two $\Gamma$-maps from $X$ to $\underline{E} \Gamma$ are homotopic through $\Gamma$-maps. If $W$ is the disjoint union of all the homogeneous spaces $\Gamma / H$, where the union is taken over all compact subgroups $H$ of $\Gamma$, then the infinite join $W * W * \cdots$ is a universal example for proper $\Gamma$-actions. If $\Gamma$ is a discrete group, there is an easier model for $\underline{E} \Gamma$. Let

$$
X_{\Gamma}=\left\{f: \Gamma \rightarrow[0,1]: f \text { has finite support and } \sum_{\gamma \in \Gamma} f(\gamma)=1\right\},
$$

equipped with the evident action of $\Gamma$ by translation and topology determined by

$$
d\left(f_{1}, f_{2}\right)=\sup _{\gamma \in \Gamma}\left|f_{1}(\gamma)-f_{2}(\gamma)\right| .
$$

Then $X_{\Gamma}$ is a proper $\Gamma$-space and is universal. As a set it is simply the geometric realization of the simplicial complex whose $q$-simplices are the $(q+1)$-element subsets of $\Gamma$.

If $\Gamma$ is a locally compact group, we then define the classifying space for proper $\Gamma$-actions to be the quotient space $\underline{B} \Gamma=\underline{E} \Gamma / \Gamma$. It is classifying in the following sense. If $X$ is any metrizable space, we say that a proper $\Gamma$-space over $X$ is a pair $(Z, p)$, where $Z$ is a proper $\Gamma$-space and $p: Z \rightarrow X$ is a continuous map such that (a) $p(g z)=p(z)$ for all $(g, z) \in \Gamma \times Z$ and (b) the map $Z / \Gamma \rightarrow X$ determined by $\Gamma$ is a homeomorphism. We say that two proper $\Gamma$-spaces $(Z, p)$ and $\left(Z^{\prime}, p^{\prime}\right)$ over $X$ are isomorphic if there is a homeomorphic $\Gamma$-map $f: Z \rightarrow Z^{\prime}$ such that $p=p^{\prime} \circ f$. If $P(\Gamma, X)$ is the set of homotopy classes of proper $\Gamma$-spaces over $X$, the function $[X, \underline{B} \Gamma] \rightarrow P(G, X)$, which assigns to the homotopy class of a map $\psi: X \rightarrow \underline{B} \Gamma$ the pullback along $\psi$ of the map $\underline{E} \Gamma \rightarrow \underline{B} \Gamma$, is a bijection of sets.

Analogous to the notion that the terminal object $E \Gamma$ in the $\Gamma$-homotopy category of $\Gamma$-CW complexes can be characterized as a free contractible $\Gamma$-space, one can use the equivariant Whitehead theorem to assert the following analogue of the wellknown nonequivariant case:

Proposition 2.1. A proper $\Gamma-C W$ complex is a model for $\underline{E} \Gamma$ iff the $H$-fixed point set $X^{H}$ is nonempty and contractible for each finite subgroup $H \subseteq \Gamma$.

In fact, one should mention that this notion can be easily generalized to account for classifying spaces of various types. Following Lück we consider a group $G$ and a family $\mathcal{F}$ of subgroups of $G$ closed under conjugation and finite intersection. A model $E(G, \mathcal{F})$ for the classifying $G-C W$ complex for $\mathcal{F}$ is a $G$-CW complex which has the properties that (i) all isotropy groups of the $G$-action on $E(G, \mathcal{F})$ belong to $\mathcal{F}$ and (ii) for any $G$-CW complex $Y$ whose isotropy groups belong to $\mathcal{F}$, there is exactly one $G$-map $Y \rightarrow X$ up to $G$-homotopy. Stated otherwise, the space $E(G, \mathcal{F})$ is a terminal object in the $G$-homotopy category of $G$-CW complexes whose isotropy groups belong to $\mathcal{F}$.

It is clear that $E(\Gamma, \mathcal{F})$ is precisely $E \Gamma$ when $\mathcal{F}=\mathcal{F}_{t}$ consists of just the trivial subgroup and that $E(\Gamma, \mathcal{F})$ is $\underline{E} \Gamma$ when $\mathcal{F}=\mathcal{F}_{c}$ is the family of compact subgroups of $\Gamma$. Of course, when $\Gamma$ is discrete, then $E\left(\Gamma, \mathcal{F}_{c}\right)$ coincides with $E\left(\Gamma, \mathcal{F}_{f}\right)$, where $\mathcal{F}_{f}$ is the family of all finite subgroups of $\Gamma$. For general $\mathcal{F}$ there is a model for $E(G, \mathcal{F})$, and any $G$-CW complex $X$ is such a model iff all of its isotropy groups belong to $\mathcal{F}$ and the fixed point set $X^{H}$ is weakly contractible for each $H \in \mathcal{F}$. 
The following examples will show that the classifying spaces $\underline{E} \Gamma$ and $\underline{B} \Gamma$ are natural objects that are oftentimes easier to compute than their free counterparts:

(1) If $\Gamma$ is compact, then any $\Gamma$-space is proper, so both $\underline{E} \Gamma$ and $\underline{B} \Gamma$ may be modelled by a single point.

(2) If $\Gamma$ has no nontrivial compact subgroups, then every proper $\Gamma$-space $X$ is a locally trivial principal $\Gamma$-bundle over $X / \Gamma$, so $\underline{E} \Gamma$ coincides with the usual universal $\Gamma$-space $E \Gamma$ and $\underline{B} \Gamma$ is identified with $B \Gamma$.

(3) Let $G$ be a Lie group with finitely many components and maximal subgroup $K$. If $\Gamma$ is any discrete subgroup of $G$, then $\underline{E} \Gamma=G / K$ and $\underline{B} \Gamma=\Gamma \backslash G / K$. If $\Gamma$ is torsion-free, then $\Gamma \backslash G / K$ is a manifold; otherwise it is an orbifold.

(4) The group $G=D_{\infty}=\mathbb{Z} \rtimes \mathbb{Z}_{2}$ acts properly on $\mathbb{Z}$, where the $\mathbb{Z}$ factor acts by translation and the $\mathbb{Z}_{2}$ factor acts by reflection across the origin. The isotropy groups are $\mathbb{Z}_{2}$ at the integers and are otherwise trivial. Therefore $\mathbb{R}$ is a model for $\underline{E} D_{\infty}$ and $\underline{B} D_{\infty}$ is a closed interval with isotropy $\mathbb{Z}_{2}$ at each endpoint.

(5) For discrete groups $\Gamma$ and $\Gamma^{\prime}$ we have $\underline{B}\left(\Gamma \times \Gamma^{\prime}\right)=\underline{B} \Gamma \times \underline{B} \Gamma^{\prime}$ and $\underline{B}\left(\Gamma * \Gamma^{\prime}\right)=$ $\underline{B} \Gamma \vee \underline{B} \Gamma^{\prime}$.

It was proved by Leary and Nucinkis 14 that, given any connected CW-complex $X$, there is a group $\Gamma$ for which $\underline{B} \Gamma$ is homotopy equivalent to $X$. Given this result, we cannot expect to simplify our analysis by exploiting any topological peculiarities intrinsic in proper classifying spaces.

\section{The Finite CASE}

For connected closed spin manifolds $M^{n}$ of dimension at least five, Jung and Stolz [11 prove that $M$ admits a positive scalar curvature metric iff the Dirac class $D([M, g])$ is represented by a spin manifold of positive scalar curvature in the real connective $K$-groups $k o_{n}(B \Gamma)$. Here $\Gamma=\pi_{1}(M)$ and $g: M \rightarrow B \Gamma$ classifies the universal cover of $M$. Our main initial tool is the corresponding result for totally nonspin manifolds, also due to Jung and Stolz. See also [10. First we offer some terminology that we will use in this section and in the following.

Definition 3.1. Let $M^{n}$ be a closed $n$-dimensional oriented manifold with fundamental group $\Gamma$. Let $g: M \rightarrow B \Gamma$ classify its universal cover. We say that $M$ is positively representable if there is a closed $n$-manifold $N$ of positive scalar curvature with fundamental group $\Gamma$ and classifying map $h: N \rightarrow B \Gamma$ such that $g_{*}[M]=h_{*}[N]$ in $H_{n}(B \Gamma)$. Here $[M]$ and $[N]$ are the fundamental classes of $M$ and $N$ in their top homology group. By abuse of terminology we may also say in this case that $[M]$ or $g_{*}[M]$ is positively representable in $H_{n}(B \Gamma)$.

Proposition 3.2 (11]). Let $M^{n}$ be a connected closed manifold of dimension $n \geq 5$ and fundamental group $\Gamma$. If $M$ is totally nonspin, then $M$ admits a positive scalar curvature metric iff $M$ is positively representable.

In effect, this theorem describes the circumstances under which the curvature phenomenon is only class-dependent. Observe, however, the point that if $\left(M^{n}, f\right)$ is a spin manifold with no positive scalar curvature metric and if $\left(N^{n}, g\right)$ is closed manifold with $\pi_{1}(M) \cong \pi_{1}(N)$ and $f_{*}[M]=g_{*}[N]$ in $H_{n}(B \Gamma, \mathbb{Z})$, then the theorem gives us no information about the curvature properties inherent in $N$. Note, however, that if $\Gamma$ is finite, Conjecture 1 predicts that every compact totally nonspin manifold is positively representable. 
Remark 3.3. Let $M$ be totally nonspin and compact. If $n=\operatorname{dim} M \geq 5$ and $H_{n}(B \Gamma)=0$, where $\Gamma=\pi_{1}(M)$, then $M$ can be endowed with a metric of positive scalar curvature. Indeed, since $H_{n}(B \Gamma)$ is trivial, the existence of any positively curved manifold $N^{n}$ with fundamental group $\Gamma$ immediately implies the existence of such a metric of $M$. One can construct such an $N$ by executing codimension $n$ and $n-1$ surgeries on the $n$-sphere $S^{n}$ with respect to the group presentation of $\Gamma$. By the surgery theorem [8, 25], this process yields a positively curved manifold.

Proposition 3.4. Conjecture 1 is true for finite cyclic groups $\mathbb{Z}_{m}$.

Proof. We will show that both halves of the conjecture hold for this particular case. Since $\mathbb{Z}_{m}$ is finite, the proper classifying space $\underline{B} \mathbb{Z}_{m}$ is a single point, and hence $H_{n}\left(\underline{B} \mathbb{Z}_{m}\right)$ is trivial for all $n \geq 1$. Hence if $M^{n}$ is a totally nonspin manifold with $n \geq 5$ and $\pi_{1}(M)=\mathbb{Z}_{m}$, then $f_{*}[M]$ must vanish in $H_{n}\left(\underline{B} \mathbb{Z}_{m}\right)$. On the other hand, we know that

$$
H_{n}\left(B \mathbb{Z}_{m}\right)= \begin{cases}\mathbb{Z} & \text { if } n=0, \\ \mathbb{Z}_{m} & \text { if } n \text { is odd, } \\ 0 & \text { otherwise. }\end{cases}
$$

If $n$ is even, then we appeal to Remark 3.3. If $n$ is odd, then it is known that $H_{n}\left(B \mathbb{Z}_{m}\right)$ is generated by the class of the $n$-dimensional Lens space $\left[L_{m}^{n} \rightarrow L_{m}^{\infty}\right]$. Since $L_{m}^{n}$ is a quotient of $S^{n}$, it is positively curved. The other classes of $H_{n}\left(B \mathbb{Z}_{m}\right)$ are represented by symmetric quotients of connected sums of $L_{m}^{n}$, so are also positively curved. The result then follows from the proposition of Jung and Stolz.

The next result incorporates the findings of Botvinnik and Rosenberg [3] and Joachim [9] in our discussion. A class in $H_{k}(B \Gamma)$ is toral if it can be represented by a map $T^{k} \rightarrow B \Gamma$. Such classes generate a subgroup denoted by $H_{k}^{\text {toral }}(B \Gamma)$. In the elementary abelian case $\Gamma=\mathbb{Z}_{p}^{r}$, the toral subgroup $H_{k}^{\text {toral }}\left(B \mathbb{Z}_{p}^{r}\right)$ is complemented by a subgroup by which we denote $H_{k}^{a t o r a l}\left(B \mathbb{Z}_{p}^{r}\right)$. For any space $X$, we denote by $R H_{*}(X)$ the image of the Thom map $\Omega_{n}^{S O}(X) \rightarrow H_{*}(X, \mathbb{Z})$ and call it representable homology.

Proposition 3.5. (1) Conjecture 1 holds for all elementary 2-groups $\mathbb{Z}_{2}^{r}$.

(2) Conjecture 2 holds for all elementary p-groups $\mathbb{Z}_{p}^{s}$ when $p$ is an odd prime.

Proof. In both of these cases we have $H_{n}(\underline{B} \pi)=0$ for all $n \geq 1$, since the fundamental groups in question are all finite.

(1) When $\Gamma=\mathbb{Z}_{2}^{r}$, then every atoral bordism class in $H_{n}(B \Gamma)$ is represented by a manifold of positive scalar curvature 3 . The same holds for all toral classes in $H_{n}(B \Gamma)$ [9. Since the collection of positively representable classes in $H_{n}(B \Gamma)$ forms a group, these combined results prove the conjecture in this case.

(2) For elementary $p$-groups $\mathbb{Z}_{p}^{r}$ with $p$ odd, every class in $R H_{n}^{\text {atoral }}(B \Gamma) \equiv$ $R H_{n}(B \Gamma) / H_{n}^{\text {toral }}(B \Gamma)$ is represented by a manifold of positive scalar curvature 3. Notice that if $n \geq s=\operatorname{rank}\left(\mathbb{Z}_{p}^{s}\right)$, then $H_{n}^{\text {toral }}(B \Gamma)$ is trivial. The resulting isomorphism $R H_{n}^{\text {atoral }}(B \Gamma) \cong R H_{n}(B \Gamma)$ gives us the desired result.

Proposition 3.6. Let $\Gamma$ be a finite group. Suppose that for all primes $p$ dividing $|\Gamma|$, Conjecture 1 holds for all Sylow p-subgroups $\Gamma_{p}$ of $\Gamma$. Then it holds for $\Gamma$. The same sort of statement holds for Conjecture 2.

Proof. Let $t_{p}: H_{n}(B \Gamma, \mathbb{Z}) \rightarrow H_{n}\left(B \Gamma_{p}, \mathbb{Z}\right)$ be the transfer map induced by the inclusion map $i_{p}: \Gamma_{p} \rightarrow \Gamma$. We use a theorem of [13] which states that a class 
$[M]$ in $H_{n}(B \Gamma, \mathbb{Z})$ is positively representable iff $t_{p}[M]$ is positively representable in $H_{n}\left(B \Gamma_{p}, \mathbb{Z}\right)$ for all primes $p$ dividing $o(G)$ and all $\Gamma_{p} \in \operatorname{Syl}_{p}(G)$. Certainly, if $M^{n}$ is a manifold satisfying the hypotheses, then as is apparent through the commutative diagram

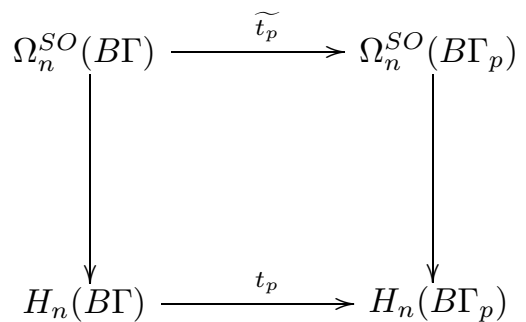

its image under the transfer $\widetilde{t_{p}}$ is positively representable, as required.

Remark 3.7. By classical work of Hölder, Burnside and Zassenhaus, a finite group all of whose Sylow subgroups are cyclic is precisely one having a group presentation of the form $\Gamma=\left\langle a, b \mid a^{m}=1=b^{n}, b^{-1} a b=a^{r}\right\rangle$, where $r^{n} \equiv 1 \bmod m, m$ is odd, $0 \leq r<m$ and $\operatorname{gcd}(m, n(r-1))=1$. See [19]. Such groups are extensions of one cyclic $p$-group by a cyclic $q$-group, and hence Conjecture 1 holds for such metacyclic groups. In particular it holds for all groups with square-free order. Statement (1) in Proposition 3.5 also verifies Conjecture 1 for the alternating groups $A_{4}$ and $A_{5}$ and any other finite group with cyclic Sylow $p$-groups for $p$ odd and elementary abelian Sylow 2-subgroups.

Remark 3.8. Clearly, Conjecture 2 holds for any group $\Gamma$ whose Sylow $p$-groups are all elementary abelian. Such groups are called elementary groups and were studied by Bechtell in 1965. They are classified as those groups $\Gamma$ for which the Frattini subgroup $\Phi(H)$ is trivial for each $H \leq \Gamma$. Equivalently, they are precisely the groups for which every normal subgroup has a complement. If a group $\Gamma$ contains a normal subgroup $K$ such that $K$ and $\Gamma / K$ are elementary groups of relatively prime order, then $\Gamma$ is elementary [2]. See [12] for various conditions under which a solvable, nilpotent or supersolvable group is elementary.

Remark 3.9. It is not known whether every toral class in $R H_{n}\left(B \mathbb{Z}_{p}^{s}\right)$ is positively representable when $p$ is odd. The belief among experts is that an affirmative answer is crucial for the Gromov-Lawson-Rosenberg conjecture to hold for general finite groups. In addition, no definitive results along these lines have been verified for groups of the form $\mathbb{Z}_{p} \times \mathbb{Z}_{p^{2}}$.

\section{The Free ABELIAN CASE}

Perhaps one of the major curiosities in the study of totally nonspin manifolds is the six-dimensional compact space $M^{6}=T^{6} \#\left(\mathbb{C P}^{2} \times S^{2}\right)$, which by minimal surface consideration lacks a metric of positive scalar curvature 25]. Conjecture 1 offers an explanation for this phenomenon. We have $\pi_{1}\left(M^{6}\right) \cong \mathbb{Z}^{6}$ and $\underline{B} \mathbb{Z}^{6}=B \mathbb{Z}^{6}$ since $\mathbb{Z}^{6}$ has no nontrivial compact factors. Most importantly, in relation to this conjecture is the fact that $f_{*}[M]=f_{*}\left[T^{6}\right]=1$ therefore represents a nonzero class in $H_{6}\left(\underline{B} \mathbb{Z}^{6}\right)$. Note that we denote by $f_{*}$ the homomorphism on groups induced by the map $M \rightarrow B \Gamma$ or the composition $M \rightarrow B \Gamma \rightarrow \underline{B} \Gamma$ as the occasion permits.

Given the above example, we may consider verifying Conjecture 1 for free abelian groups $\mathbb{Z}^{m}$. Since $\Gamma=\mathbb{Z}^{m}$ has no nontrivial compact factors, we have 
$H_{n}(\underline{B} \Gamma)=H_{n}(B \Gamma)=\mathbb{Z}^{\left(\begin{array}{c}m \\ n\end{array}\right)}$, where $\mathbb{Z}^{\left(\begin{array}{c}m \\ n\end{array}\right)}$ is understood to be trivial if $m<n$. By Remark 3.3. Conjecture 1 holds for $n$-manifolds $M^{n}$ in this range. Obstructions to positive scalar curvature metrics are difficult to identify in the general context of nonspin manifolds. A very small subset can be understood through the minimal surface techniques of Schoen and Yau, which Schick, for example, uses to provide a five-dimensional counterexample to the Gromov-Rosenberg-Lawson conjecture 24]. The following theorem and corollary provide us with the means to prove Conjecture 1 for manifolds of small dimension with free abelian fundamental group. For any space $X$ and $n \in \mathbb{Z}_{>2}$, we denote by $H_{n}^{+}(X, \mathbb{Z})$ the subgroup of $H_{n}(X, \mathbb{Z})$ consisting of all classes of the form $f_{*}[M]$, where $f: M^{n} \rightarrow X$ is a map and $M^{n}$ maintains a metric of positive scalar curvature.

Theorem 4.1 (25]). Let $M^{n}$ be a manifold endowed with a metric of positive scalar curvature with $3 \leq n \leq 7$ and let $x \in H_{n-1}(M, \mathbb{Z})$ be nonzero. Then there is a smooth orientable closed $(n-1)$-dimensional submanifold $V$ of $M$ representing $x$ such that $V$ also admits a metric of positive scalar curvature. In particular, this $V$ is a local minimum of the volume functional with trivial normal bundle.

Corollary $4.2([24)$. Let $\Gamma$ be a finitely presented group and let $n \geq 2$. Denote by $H_{n}^{+}(B \Gamma, \mathbb{Z})$ the set of $f_{*}[M] \in H_{n}(B \Gamma, \mathbb{Z})$ such that $M$ is a manifold of positive scalar curvature and $f: M \rightarrow B \Gamma$ classifies its universal cover. If $3 \leq n \leq 7$ and $\alpha \in H^{1}(B \Gamma, \mathbb{Z})$, then the homomorphism $\alpha \cap: H_{n}(B \Gamma, \mathbb{Z}) \rightarrow H_{n-1}(B \Gamma, \mathbb{Z})$ maps $H_{n}^{+}(B \Gamma, \mathbb{Z})$ into $H_{n-1}^{+}(B \Gamma, \mathbb{Z})$.

We note that these minimal surface obstructions to positive scalar curvature metrics are restricted to this range, because for manifolds of dimension 8 the minimal representatives of classes in $H_{n-1}(M)$ can have singularities in codimension 7 .

Proposition 4.3. Let $M^{n}$ be a totally nonspin manifold with fundamental group $\mathbb{Z}^{m}$, where $5 \leq n \leq 7$ and $n \leq m$. Let $f: M \rightarrow B \mathbb{Z}^{m}$ classify its universal cover. If $f_{*}[M]$ is nonzero in $H_{n}\left(B \mathbb{Z}^{m}\right)$, then $M$ cannot admit a metric of positive scalar curvature.

Proof. The proof follows the contours of the argument in [24, but we include it here for completeness. The homology class $w=f_{*}[M]$ in $H_{n}\left(B \mathbb{Z}^{m}\right)$ is an $n$-dimensional subtorus in $T^{m}=B \mathbb{Z}^{m}$. Without loss of generality, let $w=x_{1} \times \cdots \times x_{m}$, where $H_{1}\left(B \mathbb{Z}^{m}\right)=x_{1} \mathbb{Z} \oplus x_{2} \mathbb{Z} \oplus \cdots \oplus x_{m} \mathbb{Z}$. If $H^{1}\left(B \mathbb{Z}^{m}\right)=a_{1} \mathbb{Z} \oplus a_{2} \mathbb{Z} \oplus \cdots \oplus a_{m} \mathbb{Z}$, then the iterative application of cap product on $w$ by the appropriate sequence of $a_{i}$ will give a nonzero element $z=x_{m-1} \times x_{m} \in H_{2}\left(B \mathbb{Z}^{m}\right)$. Note that the 2 -sphere $S^{2}$ is the only oriented two-dimensional manifold of positive scalar curvature. Since $B \mathbb{Z}^{m}$ is aspherical, it follows that any map $g: S^{2} \rightarrow B \mathbb{Z}^{m}$ is null homotopic. In particular, we have $g_{*}\left[S^{2}\right]=0$ in $H_{2}\left(B \mathbb{Z}^{m}\right)$ for all such $g$, and so $H_{2}^{+}\left(B \mathbb{Z}^{m}\right)$ is trivial. If $M$ had positive scalar curvature, then by definition we must have $w \in H_{n}^{+}\left(B \mathbb{Z}^{m}\right)$, and so by repeated application of the above corollary we conclude that $H_{2}^{+}\left(B \mathbb{Z}^{m}\right)$ contains the nonzero element $z$, a contradiction. Notice that we have never made explicit use of the nonspin nature of $M$ in this proof.

Remark 4.4. Using a similar argument, one can extend the proposition in the same range for fundamental groups of the form $\mathbb{Z}^{r} \oplus \mathbb{Z}_{p}$, where $r$ is positive and $p \geq 2$ is prime for $n$-manifolds $M^{n}$ with $n \leq r+1$. 
Remark 4.5. Christ and Lohkamp [4] have recently announced an approach to circumvent the geometric measure theoretic problems that appear in dimensions above 7. See also [15]. Their techniques extend the minimal surface results of [25] and can be used to handle obstruction theory for positive scalar curvature metrics on enlargeable manifolds of the form $T^{n} \# N^{n}$, where $N^{n}$ is simply connected, totally nonspin and lacks a metric of positive scalar curvature. In particular, we now have at our disposal more nonspin manifolds lacking such positively curved metrics, allowing the above proposition to remain true with no upper bound on the dimension $n$.

\section{MaXimally SELF-NORMALIZING GROUPS}

We now consider the conjectures for another family of infinite groups satisfying various maximal finite subgroup conditions. Following Lück [17, 18, we say that an infinite discrete group $\Gamma$ is maximally self-normalizing if (1) every nontrivial finite subgroup of $\Gamma$ is contained in a unique maximal finite subgroup and (2) $N_{\Gamma}(M)=M$ whenever $M$ is a nontrivial maximal finite subgroup of $\Gamma$. If $\Gamma$ is maximally selfnormalizing, then one can construct a model for $\underline{E} \Gamma$ with a minimal number of cells. In particular, if $\left\{\left[M_{i}\right]\right\}_{i \in I}$ denotes the family of conjugacy classes $\left[M_{i}\right]$ of maximal finite subgroups $M_{i} \leq \Gamma$, then there is an inclusion of $\Gamma$-CW-complexes given by

$$
j: \coprod_{i \in I} \Gamma \times_{M_{i}} E M_{i} \rightarrow E \Gamma .
$$

If $u: \coprod_{i \in I} \Gamma \times_{M_{i}} E M_{i} \rightarrow \coprod_{i \in I} \Gamma / M_{i}$ is the obvious collapse map, then $\underline{E} \Gamma$ can be assembled as the $\Gamma$-pushout of the diagram

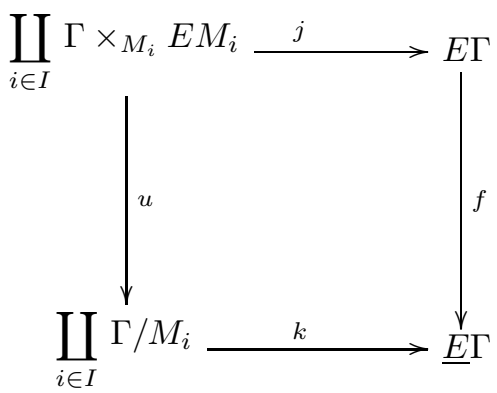

See [16] for a proof that $(\underline{E} \Gamma)^{H}$ is indeed contractible for all $H \leq \Gamma$ finite. Note that condition (2) is equivalent to the requirement that a model $\underline{E} \Gamma$ can be chosen so that the subset of points having nontrivial isotropy is discrete. In particular, these nontrivial isotropy groups are precisely the finite maximal subgroups of $\Gamma$.

The above diagram allows us to compute the group homology of $\Gamma$ from the group homology of its maximal finite subgroups. In particular, if we divide the $\Gamma$-pushout by the $\Gamma$-action, we obtain a long Mayer-Vietoris sequence given by

$$
\begin{aligned}
\cdots \longrightarrow H_{n+1}(\underline{B} \Gamma) \stackrel{\partial_{n+1}}{\longrightarrow} \bigoplus_{i \in I} \widetilde{H}_{n}\left(B M_{i}\right) \\
\stackrel{\oplus_{i \in I} H_{n}\left(B j_{i}\right)}{\longrightarrow} H_{n}(B \Gamma) \stackrel{H_{n}(\Gamma \backslash s)}{\longrightarrow} H_{n}(\underline{B} \Gamma) \stackrel{\partial_{n}}{\longrightarrow} \cdots
\end{aligned}
$$


If $\underline{E} \Gamma$ can be modelled by an $m$-dimensional complex, then $H_{n}(\underline{B} \Gamma)=0$ for $n \geq$ $m+1$. We therefore have an isomorphism $\bigoplus_{i \in I} H_{n}\left(B j_{i}\right): \bigoplus_{i \in I} \widetilde{H}_{n}\left(B M_{i}\right) \rightarrow H_{n}(B \Gamma)$ for $n \geq m+2$.

Remark 5.1. By the above isomorphism and Remark 3.3, it follows that Conjecture 2 holds when $\operatorname{dim}(\underline{E} \Gamma)<\infty$ and $\Gamma$ has no nontrivial finite subgroups.

Theorem 5.2. Let $\Gamma$ be an infinite maximally self-normalizing group for which Conjecture 1 holds for each of its finite maximal subgroups. Assume in addition that $\underline{B} \Gamma$ has the homotopy type of a $C W$ complex of dimension $\leq 3$. Then Conjecture 1 holds for $\Gamma$.

Proof. For any group $\Gamma$, we let $H_{n}^{+}(B \Gamma)$ denote the subset of $H_{n}(B \Gamma)$ represented by closed oriented $n$-manifolds of positive scalar curvature via the natural map $\Omega_{n}(B \Gamma) \rightarrow H_{n}(B \Gamma)$. This collection is in fact a subgroup (cf. [13] and [3]). Since, by hypothesis, it is assumed that $H_{n}(\underline{B} \Gamma)=0$ for $n \geq 5$, we must show that any class in the image of the natural map $\Omega_{n}(B \Gamma) \rightarrow H_{n}(B \Gamma)$ lies in $H_{n}^{+}(B \Gamma)$ for the same range of values of $n$. For all $i \in I$, let $M_{i}$ be representatives for the conjugacy classes of the maximal finite subgroups of $\Gamma$. By the Mayer-Vietoris sequence above, the map $\bigoplus_{i \in I} H_{n}\left(B j_{i}\right): \bigoplus_{i \in I} H_{n}\left(B M_{i}\right) \rightarrow H_{n}(B \Gamma)$ is an isomorphism in every dimension $n \geq 5$. Since Conjecture 1 was assumed to hold for each $M_{i}$, every representable class in $H_{n}\left(B M_{i}\right)$ lies $H_{n}^{+}\left(B M_{i}\right)$. On the other hand, we claim the representable subgroup of $H_{n}(B \Gamma)$ is precisely the image of the direct sum of the representable subgroups of the $H_{n}\left(B M_{i}\right)$. Indeed, we note that, since $\underline{B} \Gamma$ was assumed to have the homotopy type of a $\mathrm{CW}$ complex of dimension at most 3 , it follows that $\Omega_{n}(\underline{B} \Gamma)=0$ for $n \geq 5$, and so the analogue of the Mayer-Vietoris sequence in bordism gives an isomorphism from $\bigoplus_{i \in I} \Omega_{n}\left(B M_{i}\right) \rightarrow \Omega_{n}(B \Gamma)$. The result follows.

Three large families of maximally self-normalizing groups identified by Lück and Stamm [18] are enumerated as follows:

(1) Groups $G$ arising from extensions of the form $1 \rightarrow \mathbb{Z}^{n} \rightarrow G \rightarrow F \rightarrow 1$, where $F$ is finite and the conjugation action of $F$ on $\mathbb{Z}^{n}$ is free outside of $0 \in \mathbb{Z}^{n}$. The simplest nontrivial example of such an extension is the infinite dihedral group $D_{\infty}=\mathbb{Z} \rtimes \mathbb{Z}_{2}=\left\langle a, b: a^{2}, b^{2}\right\rangle$, whose maximal finite subgroups are given by $\langle a\rangle$ and $\langle b\rangle$ and their conjugates. Note that $\operatorname{dim}\left(\underline{E} D_{\infty}\right)=1$.

(2) Compact Fuchsian groups $F\left(g, r_{1}, \ldots, r_{t}\right)$ are defined by $2 g+t$ generators $a_{1}, b_{1}, \ldots, a_{g}, b_{g}, c_{1}, \ldots, c_{t}$ subject to the relations $c_{1}^{r_{1}}=\cdots=c_{t}^{r_{t}}=1$ and $\left[a_{1}, b_{1}\right] \cdots\left[a_{g}, b_{g}\right]=c_{1} \cdots c_{t}$. These groups are the discrete subgroups of $\mathrm{PSL}_{2}(\mathbb{R})$, the orientation-preserving isometries of the hyperbolic plane $\mathbb{H}^{2}$. By using the bordism theorem of Jung and Stolz for spin manifolds, Davis and Pearson [5] prove that the (unstable) Gromov-Lawson-Rosenberg conjecture holds in this case. The group $F=F\left(g, r_{1}, \ldots, r_{t}\right)$ has $t$ conjugacy classes of maximal finite subgroups, all of them cyclic. In particular, for each $i \in\{1, \ldots, t\}$ there is a singular point $v_{i} \in \mathbb{H}^{2} / \Gamma$ whose lift $\widetilde{v}_{i}$ under the branched cover $\mathbb{H}^{2} \rightarrow \mathbb{H}^{2} / \Gamma$ has stabilizer $\mathbb{Z}_{r_{i}}$. We then have $\operatorname{dim}(\underline{E} F)=2$ and

$$
H_{n}(B F) \cong \bigoplus_{i=1}^{t} \widetilde{H}_{n}\left(B \mathbb{Z}_{r_{i}}\right) .
$$


More generally, suppose that $\Gamma$ is an infinite cocompact planar group, i.e. a discontinuous group of isometries of the two-sphere $S^{2}$ or the Euclidean plane $\mathbb{R}^{2}$ or the hyperbolic plane $\mathbb{H}^{2}$ whose quotient under the $\Gamma$-action is compact. Then $\operatorname{dim}(\underline{E} \Gamma)=2$, and the maximal finite subgroups of $\Gamma$ are all cyclic or dihedral. The conjectures therefore hold for $\Gamma$ if they hold for the dihedral isotropy groups appearing in the proper classifying space.

(3) Let $\Gamma=\left\langle\left(g_{i}\right)_{i \in I}: r\right\rangle$ be a one-relator group. There is up to conjugacy a unique maximal finite subgroup $M$ of $\Gamma$, and this subgroup is cyclic. In addition there is a two-dimensional model for $\underline{E} \Gamma$ whose $\mathrm{CW}$-structure has precisely one 0-cell, one 2-cell representing the relator $r$, and as many 1-cells as there are elements of the indexing set $I$.

Example 5.3. Let $\Gamma$ be a product of $n$ infinite dihedral groups, and let $M$ be an oriented totally nonspin $n$-manifold with fundamental group $\Gamma$. By Proposition 3.5 and Theorem 5.2. Conjecture 1 is confirmed for this group. Now $\Gamma$ has a normal subgroup $\Gamma_{1}$ of finite index isomorphic to $\mathbb{Z}^{n}$. Let $N$ be the cover of $M$ corresponding to $\Gamma_{1}$. But by properties (4) and (5) in the discussion following Proposition 2.1, the proper classifying space $\underline{B} \Gamma$ is contractible. If Conjecture 1 is correct, then $M$ should admit a metric of positive scalar curvature, which then lifts to such a metric on the cover $N$. At first glance, however, one may suspect that the obvious map from the $n$-manifold $N$ to the $n$-torus $T^{n}=B \mathbb{Z}^{n}$ provides an obstruction to positive scalar curvature, but one can easily show that the obstruction for the cover always vanishes. Indeed, let $f_{*}: H_{n}(M) \rightarrow H_{n}(B \Gamma)$ be the natural map on homology and let $g_{*}: H_{n}(N) \rightarrow H_{n}\left(B \Gamma_{1}\right) \cong H_{n}\left(T^{n}\right)$ be the lifted map on the cover. Let $t_{*}: H_{n}(B \Gamma) \rightarrow H_{n}\left(B \Gamma_{1}\right)$ be the transfer map. Then $g_{*}[N]=t_{*} f_{*}[M]$ in $H_{n}\left(B \mathbb{Z}^{n}\right)$. However, there is a natural action of $\mathbb{Z}_{2}^{n}$ on $H_{n}\left(B \mathbb{Z}^{n}\right)$ and $t_{*} f_{*}[M]$ belongs to the fixed set of this action; but the fixed set is trivial, so the obstruction vanishes. In more general settings with lifts corresponding to finite covers $\Gamma_{1} \rightarrow \Gamma$, one can appeal generalized transfer functions to obtain similar results; see [7.

Remark 5.4. Consult [16] for conditions for which a discrete group $\Gamma$ has a $d$ dimensional model for $E \Gamma$. For example, Dunwoody 6 proves that there is a 1-dimensional model for $\underline{E} \Gamma$ iff the rational cohomological dimension of $\Gamma$ is at most 1 .

Remark 5.5. Conjectures 1 and 2 have been additionally verified by the author for some $\Gamma$ whose group homologies are well known or whose proper classifying spaces exhibit tractable isotropy (certain $p$-groups, triangle groups, the lamplighter group, certain free products, and groups acting on certain trees), and the underlying proofs run in the same vein as for Proposition 3.6 and Theorem 5.2. The methods of this paper fail to extend, for example, to generalized reflection or Coxeter groups $\Gamma$, for which the points in $\underline{B} \Gamma$ carrying nontrivial isotropy are not isolated but instead form a subcomplex of nonzero dimension. We hope that the development of additional homological tools or new ways of constructing nonspin manifolds without positive scalar curvature will settle the conjectures either positively or negatively.

Remark 5.6. The referee has offered the following insight. A parallel theory for positive scalar curvature for nonorientable manifolds has been developed, the analogues of Definitions 3.1 and Proposition 3.2 having been established for this case (see [3]). Instead of using ordinary homology with integer coefficients, one simply considers $H_{n}(, w)$, i.e. homology with coefficients in the local coefficient system 
locally given by the integers but twisted by $w=w_{1}: \pi_{1}(M) \rightarrow \mathbb{Z}_{2}$, the first StiefelWhitney class of $M$. Any conjecture about the positive scalar curvature problem in the totally nonspin case should therefore appear with a twisted version. The impediment is that, while $H_{n}(B \Gamma, w)$ makes sense, the object $H_{n}(\underline{B} \Gamma, w)$ may not, since $w$ may not factor through the map $\Gamma=\pi_{1}(B \Gamma) \rightarrow \pi_{1}(\underline{B} \Gamma)$. One possible way to circumvent this difficulty is to modify the definition of $\underline{B} \Gamma$ by constructing instead a terminal object $\underline{B} \Gamma^{w}$ in terms of classifiying spaces for a family of subgroups (see [16]). Instead of using all finite subgroups, perhaps one should use all finite subgroups satisfying a particular $w_{1}$ condition. At the moment, however, there do not seem to be sufficiently many developed examples to generate a credible conjecture that provides a precise obstruction for positive scalar curvature in this case. We hope that future research will provide the motivation to bring insight to this interesting situation.

\section{REFERENCES}

[1] P. Baum, A. Connes and N. Higson, Classifying space for proper actions and K-theory of group $C^{*}$-algebras, $C^{*}$-algebras: 1943-1993 (San Antonio, TX, 1993), 240-291, Contemp. Math., 167, Amer. Math. Soc., Providence, RI, 1994. MR.1292018 (96c:46070)

[2] H. Bechtell, Elementary groups, Trans. Amer. Math. Soc. 114 (1965), 355-362. MR0175967 $(31: 243)$

[3] B. Botvinnik and J. Rosenberg, The Yamabe invariant for non-simply connected manifolds, J. Differential Geom. 62 (2002), no. 2, 175-208. MR1988502 (2004j:53045)

[4] U. Christ and J. Lohkamp, Singular minimal hypersurfaces and scalar curvature, preprint, arXiv:math/0609338.

[5] J. Davis and K. Pearson, The Gromov-Lawson-Rosenberg conjecture for cocompact Fuchsian groups, Proc. Amer. Math. Soc. 131 (2003), no. 11, 3571-3578 (electronic). MR.1991770 (2004f:53039)

[6] M. Dunwoody, Accessibility and groups of cohomological dimension one, Proc. London Math. Soc. (3) 38 (1979), no. 2, 193-215. MR531159 (80i:20024)

[7] B. Eckmann, Cohomology of groups and transfer, Ann. of Math. (2) 58 (1953), 481-493. MR0058600 (15:397a)

[8] M. Gromov and B. Lawson, The classification of simply connected manifolds of positive scalar curvature, Annals of Mathematics 111 (1980), 423-434. MR.577131(81h:53036)

[9] M. Joachim, Toral classes and the Gromov-Lawson-Rosenberg conjecture for elementary abelian 2-groups, Arch. Math. (Basel) 83 (2004), no. 5, 461-466. MR2102644 (2005g:53050)

[10] M. Joachim and T. Schick, Positive and negative results concerning the Gromov-LawsonRosenberg conjecture, Geometry and topology: Aarhus (1998), 213-226, Contemp. Math., 258, Amer. Math. Soc., Providence, RI, 2000. MR.1778107 (2002g:53079)

[11] R. Jung and S. Stolz, private communication.

[12] L. Kappe and J. Kirtland, Finite groups with trivial Frattini subgroup, Arch. Math. (Basel) 80 (2003), no. 3, 225-234. MR.1981175 (2004f:20046)

[13] S. Kwasik and R. Schultz, Positive scalar curvature and periodic fundamental groups, Comment. Math. Helv. 65 (1990), no. 2, 271-286. MR.1057244 (91k:57027)

[14] I. Leary and B. Nucinkis, Every $C W$-complex is a classifying space for proper bundles, Topology 40 (2001), no. 3, 539-550. MR1838994 (2002c:55022)

[15] J. Lohkamp, Positive scalar curvature in dim $\geq 8$, C. R. Math. Acad. Sci. Paris 343 (2006), no. 9, 585-588. MR.2269869 (2008d:53079)

[16] W. Lück, Survey on classifying spaces for families of subgroups, Infinite groups: Geometric, combinatorial and dynamical aspects, 269-322, Progr. Math., 248, Birkhäuser, Basel, 2005. MR2195456 (2006m:55036)

[17] W. Lück, The type of the classifying space for a family of subgroups, J. Pure Appl. Algebra 149 (2000), no. 2, 177-203. MR1757730 (2001i:55018)

[18] W. Lück and R. Stamm, Computations of $K$ - and L-theory of cocompact planar groups, $K$-Theory 21 (2000), no. 3, 249-292. MR.1803230 (2001k:19004) 
[19] D. Robinson, A course in the theory of groups, second edition, Graduate Texts in Mathematics, 80, Springer-Verlag, New York, 1996. MR1357169 (96f:20001)

[20] J. Rosenberg, $C^{*}$-algebras, positive scalar curvature, and the Novikov conjecture, Inst. Hautes Études Sci. Publ. Math., no. 58 (1983), 197-212. MR720934 (85g:58083)

[21] J. Rosenberg, $C^{*}$-algebras, positive scalar curvature and the Novikov conjecture. II, Geometric methods in operator algebras (Kyoto, 1983), 341-374, Pitman Res. Notes Math. Ser., 123, Longman Sci. Tech., Harlow, 1986. MR866507 (88f:58140)

[22] J. Rosenberg, $C^{*}$-algebras, positive scalar curvature, and the Novikov conjecture. III, Topology 25 (1986), no. 3, 319-336. MR842428 (88f:58141)

[23] J. Rosenberg and S. Stolz, A "stable" version of the Gromov-Lawson conjecture, The Čech centennial (Boston, MA, 1993), 405-418, Contemp. Math., 181, Amer. Math. Soc., Providence, RI, 1995. MR1321004 (96m:53042)

[24] T. Schick, A counterexample to the (unstable) Gromov-Lawson-Rosenberg conjecture, Topology 37 (1998), no. 6, 1165-1168. MR1632971 (99j:53049)

[25] R. Schoen and S.-T. Yau, On the structure of manifolds with positive scalar curvature, Manuscripta Math. 28 (1979), no. 1-3, 159-183. MR535700 (80k:53064)

[26] R. Schultz, Positive scalar curvature and odd order abelian fundamental groups, Proc. Amer. Math. Soc. 125 (1997), no. 3, 907-915. MR.1363184 (97j:53041)

Department of Mathematics, Wellesley College, Wellesley, Massachusetts 02481

E-mail address: schang@wellesley.edu 